Portland State University

PDXScholar

Systems Science Faculty Publications and

Presentations

Systems Science

Summer 6-22-2021

\title{
Universal Biological Motions for Educational Robot Theatre and Games
}

\author{
Rajesh Venkatachalapathy \\ Portland State University, venkatr@pdx.edu \\ Martin Zwick \\ Portland State University, zwick@pdx.edu \\ Adam Slowik \\ Koszalin University of Technology \\ Kai Brooks \\ Portland State University \\ Mikhail Mayers \\ Portland State University
}

See next page for additional authors

Follow this and additional works at: https://pdxscholar.library.pdx.edu/sysc_fac

Part of the Computer Sciences Commons, Electrical and Computer Engineering Commons, Engineering Education Commons, and the Non-linear Dynamics Commons Let us know how access to this document benefits you.

\section{Citation Details}

R. Venkatachalapathy et al. (2021). "Universal Biological Motions for Educational Robot Theatre and Games," 2021 International Conference on Information and Digital Technologies (IDT), pp. 162-170, doi: 10.1109/IDT52577.2021.9497592.

This Post-Print is brought to you for free and open access. It has been accepted for inclusion in Systems Science Faculty Publications and Presentations by an authorized administrator of PDXScholar. Please contact us if we can make this document more accessible: pdxscholar@pdx.edu. 


\section{Authors}

Rajesh Venkatachalapathy, Martin Zwick, Adam Slowik, Kai Brooks, Mikhail Mayers, Roman Minko, Tyler Hull, Bliss Brass, and Marek Perkowski 


\section{Universal Biological Motions for Educational Robot Theatre and Games}

\author{
Rajesh Venkatachalapathy, \\ Martin Zwick \\ System Science, Portland State \\ University \\ Portland, Oregon, USA
}

\author{
Adam Slowik \\ Department of Electronics and \\ Computer Science \\ Koszalin University of Technology \\ Koszalin, Poland
}

\author{
Kai Brooks, Mikhail Mayers, Roman Minko, \\ Tyler Hull, Bliss Brass, Marek Perkowski \\ Department of Electrical and Computer \\ Engineering \\ Portland State University \\ Portland, Oregon, USA
}

\begin{abstract}
Paper presents a concept that is new to robotics education and social robotics. It is based on theatrical games, in which students create "biological", "characteristic" and natural motions for social robots and animatronic robots. Presented here motion model is based on Drift Differential Model from biology and Fokker-Planck equations. This model is used in various areas of science to describe many types of motion. The model was successfully verified on various simulated mobile robots and a motion game of three robots called "Mouse and Cheese".
\end{abstract}

\section{INTRODUCTION. PROBLEM FORMULATION}

Games are already an important aspect of modern educational efforts on the levels of high school and undergraduate education because teenagers and young people are enthusiastic about playing and programming games. As an example, paper [41] presents how games can be used to teach logic minimization, circuit design and quantum mechanics. Paper [1] discusses video games in this context, while paper [3] and a comprehensive book [2] present various approaches and ideas of designing video games for education. Different types of games are also used in school theatres to train acting skills [4, 5, 9]. The robot social games are used to help autistic children gain interpersonal skills [7]. Many ideas about didactic and realization aspects of robot theatre are in [6], while [8] discusses the idea of combining games and robots in computer science education. An ambitious task of using robot theatre to promote STEM education for underserved students is discussed in [10].

In addition, robots are increasingly used to teach children, teenagers and university students about mechanical and electrical hardware design, programming, mathematics, gaming and humaninteraction skills. Moreover, humanoid and animatronic robots are already used in several areas of human-robot interaction: (1) to interact with elderly and autistic children, (2) as robot museum guides and receptionists, (3) as home robots and popular toys, (4) as robot entertainers. Since 2001 the last author, Marek Perkowski, teaches teenagers about robotics, logic, mathematics and systems science approaches. One of the ways to achieve these educational goals is through creating an improvisational robot theatre that combines the ideas of teaching through robotics, through theatre and through games.

One of important problems in educational robotics is designing the motions of the robot that would be natural: human-like, or specific animal-like. This is also related to the art of animation in puppet theatre, movies and computer games. In robot theatre the motions can be not necessarily human-like but must be "characteristic and believable" or "biological". How to program an inexpensive robot built from an internet kit to "run like a dog"? To "eat like a nutria"?

As a more advanced example, let us discuss a hand-waving greeting gesture "hello" by a humanoid robot that uses one or two arms for his emotional gestures. If the greeting action would be always the same, the robot would be perceived as boring and unnatural by people who interact with this robot or watch the robot theatre performance. Simple solution to this problem is to design several greeting motions and select one randomly unfortunately we observed in our many robot theatrical plays that this solution also becomes unnatural after few minutes of watching these repeated behaviors. Another solution may be to design a greeting motion as a sequence of completely random poses. These motions are quite unrealistic and also become not interesting soon.

Thus, several research directions have been tried to create interesting human-like and varying gestures for animatronic and humanoid robots. They include: (1) robot programming languages based on elementary motions and algebraic operators to combine them [12], (2) low-level robot motion editors to design motions (gestures) as sequences of postures (similar to graphic animations), (3) methods based on feedback from position sensors, (4) human motion acquisition and transformation to robot kinematics and dynamics, (5) heuristic systems for converting music to motions, (6) random number generators, (7) Machine Learning approaches, (8) methods based on spectral analysis of individual motion waves from sensors, (9) Perlin noise, and several others $[20,12,29,4544,38,37,14]$.

These methods and their extensions are used to create complex robot behaviors, which involve also simulated emotions and conversations in natural language. Each of these methods creates better or worse typical examples and variants of motions and behaviors, but as evaluated by psychologists, puppeteers, animators and theatre experts [12], the methods still do not satisfy the criteria of robot's sufficient theatrical realism, which level has been already achieved in the technical areas of graphic animations and games [12], and of course in the art of puppetry. No objective methods exist for the evaluation of behavior quality of such robots.

The problems that we address in this paper are the following:

(1) We outline a new model: realistic ("biological") motion generation problem, based on general principles of motions in Nature. 
(2) We present an interesting issue for robot theatre: "designing new motions for animatronic, humanoid and other theatrical and social robots". Should they have motions similar to humans? Similar to machines? To animals? To Fairy Tale Characters? To electrons in an atom? Attempt to design motions that are new and unexpected, interesting, symbolic, information-carrying and characteristic are important for improvisational robot theatre.

(3) We create a model of motions for robots playing motionbehavior games, like policemen chasing a thief or an airplane battle. The motions may be influenced by noise, have controllable errors or some other parameters so that the motions are not predictable by opponents but are interesting to watch by the game audience (our games are a kind of interactive, improvisational theatres, like a cheese escaping from hungry mice and asking verbally for mercy).

New methods to solve these three highly related problems should be thus developed, implemented, evaluated, and compared with the existing methods. This paper presents our attempt at creating such a new method for game-base improvisational educational robot theatres, distinct from all previous approaches known from the literature.

\section{PREVIOUS RESEARCH}

When we create improvisational robot theatre for education we want to base our thinking on system approaches that can solve wide categories of problems and have also deep educational aspects. These issues are discussed with teen teams while solving sequences of design examples, math problems, and programming exercises. Such system approach can be justified also on philosophical, conceptual and aesthetic grounds; it can also be a part of conventional scientific discovery processes. Different perspectives on this process have been offered [30, 37, 25, 22]. Hofstadter emphasizes the role of analogical reasoning in scientific discovery. Analogy is used in SAT tests and plays also a fundamental role in children theatre. Simon [37] offers a more computational approach and works from a discovery of knowledge as a foraging process (foraging is a common topic in plays about animals). Holland and Thagard build on this and the concept of coherence to develop a model of induction. Modern enterprises like Machine Learning find transfer learning to be a key concept underpinning many useful artificial systems.

Taken together, these models of scientific discovery require search and recognition heuristics. The models are meant to capture consistency criteria among scientific explanations, theory induction and other steps during scientific knowledge accumulation [25]. Such heuristics allow a transfer of concepts and models from one domain to another. In this paper, with the goal of creating educational robots and robot team behaviors, we identify these isomorphisms in certain classes of stochastic dynamical systems, we construct a notion of reliability as satisfaction of constraints, and we express them as first-passage time to a boundary (FPT). The probability distribution of the first exit out of a region in state space has a natural bio-semiotic interpretation, which in our case is applied to escaping and reaching robot motions in games and educational plays. Running, following, escaping and reaching actions are very common in all puppet plays and computer games. These proposed by us system-based methods have been however never used to generate robot behaviors. This seems to be the most important contribution of this paper from the theoretical point of view, while the practical aspect of our work is designing concrete realistic motions for our theatrical robots.

The biggest issue with the pre-programmed robot motion libraries for entertainment robots is that the robots can quickly become unnatural and boring to their audiences. This is because often there are only a few ready motions in the memorized motion library, and there are only few circumstances that the robot can respond to. Thus, the results are seemingly repetitive robot responses and the perception of limited interaction. Researchers in the animation field try to find ways to enable real-time response generation for virtual agents (e.g. video game characters). Bruderlin and Williams showed that by representing motion data as signals, common signal processing techniques such as multiresolution filtering, Fourier Analysis, wave shaping, time warping, and interpolation can be applied to the motion data [23]. As signals, motion data can be manipulated in real-time to be exaggerated, subdued, or blended with other motions while maintaining the characteristics of the original motion. Unuma et al. used Fourier Analysis to create transitions between two periodic motions using normalized coefficients $[0,1]$ between the Fourier coefficients of the two motions [24]. Also, Unuma showed by using Fourier Analysis, characteristic functions (e.g. 'tiredness') can be extracted by calculating the difference between the coefficients of a 'neutral' motion (e.g. walk), and its variation (e.g. tired walk). The extracted characteristic function can then be applied to other motion (e.g. run) to create a similar characteristic on the other motion (e.g. tired run).

Ken Perlin developed a method to generate pseudo-random noises that can be used for creating noise in animation (onedimension) to animated solid textures (four-dimensions) [20]. Perlin's noise-generating method (popularly known as Perlin's Noise) have been used to create noise in the movement of a virtual character or robot, to simulate those little movements such as breathing, blinking, fidgeting, or sways. Originally, Perlin Noise is often used to create and animate movements of textures in nature such as water, clouds, fire, and other elements. Perlin Noise is generated by creating a sequence of random noises. The sequence of noise usually starts with a smoothed (i.e. interpolated) low frequency noise to $n$ number of higher frequency noise, where each subsequent noise frequency is an octave higher than the last $(f n=2 * f n-1)$. The sequence of noises is then added together; with the contribution of each random noise decreasing as the frequency of the noise signal increases (noises with higher octave have less contribution than the lower octave noises to the final Perlin's Noise). The Perlin's Noise is especially useful to alleviate the 'static look' when a virtual character or robot is idle; instead of being still without any movement, the little movements (i.e. noise) give an impression of breathing or heartbeats, thus giving the illusion that the agent is 'alive.' Our approach is based on a similar philosophy of creating a special type of "noise", but we base the motions on Fokker Planck differential equations. Thus in addition to Perlin's noise the "Fokker-Planck Noise" is used to create motions for "fairy tale robot actors". 


\section{New Methodology BASEd ON UnIVERSAl Motions, STOCHASTIC DYNAMICAL SYSTEMS AND FINE-TUNING}

\section{A. Motions and Emotions of theatrical robots}

The motion of a social/theatrical robot should be realistic, interesting, human-like (animal-like, fairy-tale-like or robot-like), and demonstrating some specific "robot personality" and "robot emotion". The motions/behaviors creating method should produce stochastic variants to create very many unpredictable but similar motions, because this makes the robot more interesting and increases its repertoire of behaviors in games. On the other hand, the motion must be robust; which means that with high probability "strange and out of place" motions will not be generated by the stochastic motion generator. We can thus say that the motion-generating mechanism must be reliable. For instance, the motion of a small robot escaping from a light beam should be not programmed. This robot should behave always differently, but in principle it should be able to escape to a safe dark space at least in some cases. Similarly, a robot arm reaching for an apple located above should be ultimately able to grasp the apple. The generated motion should be also related to the environment: a robot control mechanism for "dancing waltz" should have always the same basic pattern, but the actual motion is different for a robot in an open area and a robot dancing in a narrow corridor.

Environment is responsible for some additional constraints in time, space or robot behavior type. As an example, a robot with 22 degrees of freedom (such as rotations in head, arms and knees) can be treated as a dynamic system that solves in real time the set of differential equations with at least 22 variables. These equations can be separate; in the simplest case we have a system of synchronized 22 one-variable differential equations. Parameters of these equations come from robot's memory (like emotional state of the robot, robot being happy or sad - the internal state of a hybrid state machine) or from the environment (like a distance of robot to a wall). In general, the robot-related variables in these differential equations can represent: spatial coordinates, velocities, radial velocities, accelerations, forces, rotation angles, emotions or sensor readings.

It is known from puppet theatre studies that puppet's motion is the most important artistic and educational aspect for very young audiences. Trying to develop a fundamental philosophy for robot theatres, we may ask ${ }_{2}$ what are the most general motions that exist in the world? The linear motion and the circular motion come immediately to mind, as they are solutions of simple differential equations and are common: the motion of planets around the Sun, the motion of electrons in the atom. Diffusion processes are another motions that occur in biology, sociology and psychology $[11,17,18,19,21,26]$. Thus Fokker-Planck and Schrödinger equations come immediately to our mind as universal motion generators. What else? We assume that for a robot theatre one should dispose a system that could generate all basic motions that exist in the world, including cosmic and atomic motions. These types of motions are not only entertaining but have also important educational aspects.

\section{B. Universal motions from differential equations.}

One can say that mathematically a robot is a dynamic system of differential equations. Because of the above-mentioned requirements of realism, universality and interestingness, our preferred model is that of stochastic differential equations.

The questions arise:

(1) What equations and on what variables?

(2) How these equations are solved?

(3) How to model noise, slippage and hardware imperfections or even errors?

Differential equations are (together with Cellular Automata) two of the most general mathematical descriptions of system dynamics and problem-solving. They are used in physics, chemistry, biology, psychology and sociology, to list just a few. They describe dynamics of linear and non-linear systems, including systems based on agents. Agents can be robots or their subsystems. Although stochastic differential systems (SDS) are common in robotics, they have been not yet applied to any of the three groups of problems given in section I. The main idea of our research is to apply general differential equation types from various research areas, such as physics, chemistry, biology, psychology and sociology, to create interesting but reliable motions for theatrical robots. While [26] concentrates on models from biology, sociology and psychology, only a small subset of model equations from [26] will be illustrated in this paper.

\section{Biological, psychological and social agents.}

One can observe a surprisingly deterministic behavior of biological or social agents in the presence of stochasticity. Notable is the reliability of this behavior. The general nature of this behavior requires a study centered on general principles. This makes it an ideal candidate for a systems approach to various processes abstracted as motions. After constructing a specific notion of reliability, we find isomorphisms between biological, social and psychological processes [26] that demonstrate how the same notion of reliability developed in [26] can be used in biology for animal motions, in sociology to understand escape from poverty traps, and in psychology to understand decision making.

Along the way, we discover and discuss connections within related sub-fields in biology, sociology and psychology, illustrating the utility of system approach in scientific inquiry and mathematical exploration, with direct application to universal robot motion generation. This approach is useful mostly for humanoid assistive, theatrical, social and game-playing robots, but for simplification in this paper we present three-wheeled small mobile robots called Braitenberg Vehicles [32]. This is because of: (1) they can be built in short time even by young teenagers from inexpensive kits that many are advertised on Amazon, eBay and internet, (2) they have the simplest possible kinematics which simplifies programming of motions, (2) they allow for easy creation of robot swarms with interesting and non-trivial behaviors.

We formulate a hypothesis that some basic dynamic systems with their differential equations are common in many various areas - we can call them system-level motion isomorphisms. Such systems are treated as describing general motions - a holistic idea that "motions in various areas represent some characteristic patterns". These patterns are then used by us as a new methodology for robot motion generation. These model similarities are the system isomorphisms: a trajectory of robot trying to avoid obstacles on his way to the closest door is similar 
to a status/education/wealth trajectory of a human rising from poverty traps. Can we apply the same differential equations to describe the dynamics of both systems?

In the highly biology-centered perspective [48], the conceptual aspect is based on an assortment of ideas emphasizing biological semantics and the biological level of motions. It carries with it a general biology-like semantics. The mathematical level that is next used for mobile robot programming is more abstract than the conceptual aspect with no semantics associated with it. By lifting a scientific concept and treating it as a system concept, models associated with the original scientific concept are available to be used in disciplinary scientific inquiry in fields unrelated to the original scientific concept, robot motion theory and game applications in our case. The micro- and nano- scale physiological processes are modeled by a certain class of SDS. First-exits or first-passage times of these SDS are adequate descriptors of transient characteristics of these biological processes [23]. This is then used in [26] for scientific inquiry in sociology and psychology. For instance: (1) in sociology, the concept of reliability is associated with dynamics in and out of poverty traps (2) in psychology, the concept of reliability is associated with dynamic judgment and decision tasks. These systems dynamics are already used in theatres and can be used in robot theatre for symbolic motions.

The isomorphism between these two processes extends to the notion of fine-tuning and constraint satisfaction, both lifted from physiology [26]. Constraint satisfaction methods are already used in robotics and Fokker-Planck equations are already used in biology, but we believe that the system analogies of biological, sociological, psychological, financial and other "motions" can find even deeper applications in designing behaviors and teaching of several types of futuristic robots in future improvisational interactive theatres. The difference of our approach is that while other authors want to analyze Fokker-Planck equations to avoid effects of noise in robotics, we want to add several special types of "noise" for theatrical and game-playing effects.

\section{Studying Animal Behaviors}

Tinbergen's vision for ethology [40] is a good starting point for researching principles of animal behavior. His "Four Questions" are:

(1) what is its physiological causation?

(2) what is its function or survival value?

(3) how has it evolved over time?

(4) how has it developed in the individual?

Shettleworth [11] summarizes these four questions to be about cause, function, evolution and development respectively and added fifth question on intra-level and inter-level integration of different disciplines answering these "Four Questions". Webb [13] expands the discussion to include behavior of artificial agents and is related to an early historical work by Brooks [15], a paper that caused a break-through and initiated a new wave of research in robotics. And finally, focusing on higher organisms, Bullock's work on comparative neuro-ethology [16] emphasizes diversity of neural mechanisms that generate behavior. This can in turn find applications in "group robotics", social robotics and "improvisational robot theatre" [12, 35, 36]. Animal behavior is typically documented using ethograms [27], recordings of behavioral repertoires decomposed into behavioral primitives. An understanding of these primitives is of general interest to ethologists. Animal behaviors can be captured the same way as human motion is acquired and used to control "biological" and humanoid robot behaviors [38]. These behavioral primitives can be mathematically characterized, modeled, transformed and generated $[12,13]$. Among the various observed characteristics, a behavior's reliability is the core focus of this paper. And this reliability is often defined by time-to-event constraints that need to be satisfied in the presence of stochasticity. Here we restrict ourselves to two such primitives: Escape behavior is evasion in response to typically harmful environmental signal. Startle

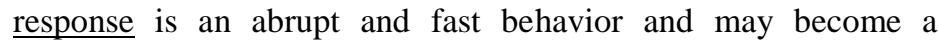
building block for more complex startles. From a modeling point of view, we can ignore the subtle differences between escapes and startles and in what follows focus on startles, treating escapes to be conceptually synonymous. Fixed action patterns, on the other hand, are also triggered by environmental cues, but are spatiotemporally complex and longer timescale behaviors. It should be pointed out that our goal is not only to build an interesting robot theatre with teens, but we also want to teach them mathematics (Fokker-Planck), biology (FP in animal motions), programming, game theory and understanding the relation between abstract differential equations and other models and actual motion of the robot related to physical, mechanical design and control aspects such as noise and slipping.

\section{E. Fixed Action Patterns, Gaits and characteristic behaviors}

Consider an organism with or without a nervous system whose body size and weight are small enough so that environmental fluctuations induce instability in the organism's movement. Suppose the organism is exposed to sudden harmful stimulus. In response to this environmental signal, the organism must reliably escape or respond to avoid consequences. Adaptation to exposure of harmful signals may occur evolutionarily, developmentally or over its life history. Similarly, consider a situation where the organism, upon presentation of a cue, is expected to locomote in space. This locomotion, a fixed action pattern $(\boldsymbol{F A P})$, requires the system repeat its body configurations over an extended but finite period of time, in a stochastic environment. The disturbance may wobble and destabilize locomotion but not by much. FAP is a fundamental method in simple walking robots such as hexapods. In the case of escape and startles, ideal behavior is one that completes the act by a definite time. In the case of FAP, ideal behavior is one that maintains the pattern for a definite time. Both primitives involve time constants; in case of startles and escape, it is time to viability; in the case of FAP, it is persistence of viability. Typically, both these kinds of behaviors are modeled either using optimality principles or are built ad-hoc to reproduce behavioral templates. A key question is the source of reliable behavior under varying conditions of internal and external stochasticity. Reliable startle behavior involves movement of the body of the organism in an environment; it involves avoiding a subset of space of possible configurations while reaching a subset of final configurations within a fixed time. Reliable fixed action pattern involves repetitive movement of the body in an environment, while being subjected to uncertainty in conditions both internal and external to the organism. The notion of reliability is usually given an average case interpretation. According to this, behavior is reliable if it performs well most of the time. Behavior is reliable if we can guarantee that the agent's state is viable, even in the worst-case. Also, reverse engineering 
explanations make use of control-theory-based modules to explain reliability. Instead, we find constraint-satisfaction-based reliability guarantees more appealing. Our focus on dynamic systems reliability can be justified biologically [16], and it can also be justified by an argument similar to that of Brooks [15], who denied the need for representation in robot control. One can instead think of behavior as being generated by making use of internal representations. One potential remedial strategy might be to think of behavior as being generated by merely a biological substrate. One can call such a lower form of internal process physiological, as opposed to cognitive; and can correspondingly consider the dynamic systems type of reliability discussed in this paper as also lower than the type of reliability that is achieved via control modules. Our paper does not seek to replace the cognitive; it merely suggests that some aspects of control can be replaced with a lower form of behavior. An interesting example is the crawling locomotion of soft-bodied animals [33]. Such repetitive behavior is usually generated by central pattern generators $(\boldsymbol{C P G})$. A neuro-mechanical model that enables rhythmic movement by coupling the neuro-muscular dynamics with the environment, via friction generated by the body, is able to generate full organism coordination of patterned proprioceptive crawling. In this example, even if nervous system is involved, the locomotion is not due to precisely controlled neural circuits. It is because of fine tuning of parameters of the coupled dynamics. This example illustrates one of the main points of the paper: fine tuning with or without constraint satisfaction - under some circumstances - replaces the need for advanced control modules.

\section{MOtION GENERATION FOR ACTORS OF THE "MicE AND CHEese GAME"}

\section{A. Control of Actors - the Braitenberg Vehicles}

Although our methods are predominantly for robots or swarms with many degrees of freedom (system variables) here for simplicity we describe a simple mobile robot with two sensors, two wheels and two motors. Such robots are used much in our research and theatres [41] and we illustrate many concepts and design procedures with them [36]. This model of kinematics is better than a car or tank models in case of social and theatrical robots. All these robots start from the concept of the so-called Braitenberg Vehicles, presented in a book [32] which deeply influenced Brook's approach [15] to robot design and control.

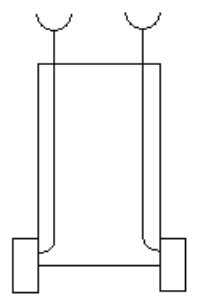

a

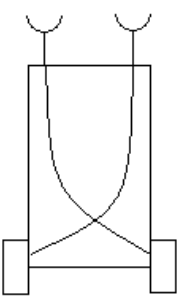

b

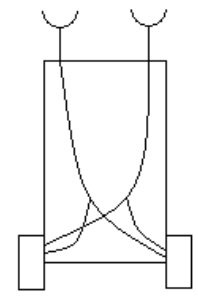

c
Fig. 1. The simplest Braitenberg Vehicles with analog control, (a) each sensor $i$ connected to the motor on the same side, $(b)$ each sensor connected to the motor on opposite side, $(c)$ both sensors connected to both the motors.

Valentino Braitenberg wrote a revolutionary book [32]. In this book he describes a series of thought experiments. It is shown in these experiments how simple systems (the vehicles, our robots) can display complex life-like behaviors far beyond those which would be expected from the simple structure of their 'brains'. He describes the law called the "law of uphill analysis and downhill invention". The law explains that it is far easier to create machines that exhibit complex behavior than it is to try and build the structures from the behavioral observations. By connecting simple motors to sensors, crossing wires and making some of them inhibitory, we can construct simple robots that could demonstrate behaviors similar to fear, aggression, love, affection, and other.

Original Braitenberg vehicles use just analog signals or Boolean logic, but we have generalized the controllers that exist between sensors and effectors to multiple-valued, fuzzy, probabilistic and quantum logics. Also by adding memory to these combinational logias, our controllers are generalized to state machines. Finally, we create hybrid automata controllers. These automata used in our paper can be treated as standard Moore automata with every internal state corresponding to certain motion, behavior type or emotion. The input states come from sensors (vision). Outputs are motion signals such as velocities given to wheels. Some set of differential equations (such as Fokker-Planck) correspond to every internal state. They generate wheel velocities for each wheel individually. Thus our hybrid automata are more general than standard automata because in standard automaton we have a constant output signal and in hybrid automaton we have a dynamical process that continues until the automaton remains in its given internal state.

Let us explain what we understand by robot emotions and behaviors. The vehicles from Fig. 1 have two sensors and two motors, right and left. These vehicles can be controlled by the way the sensors are connected to the motors. Braitenberg defines three different basic ways we could possibly connect the two sensors to the two motors: (1) Each sensor connected to the motor on the same side, (2) Each sensor connected to the motor on the opposite side, (3) Both sensors connected to both the motors. Type (1) vehicle will spend more time in places where there is less of the stuff that excites its sensors and will speed up when it is exposed to higher concentrations. If the source of the light (for light sensors) is directly ahead, the vehicle may hit the source unless it is deflected from its course. If the source is to one side, one of the sensors, the one nearer to the source, is excited more than the other. The corresponding motor turns faster. As a consequence, the vehicle will turn away from the source. Turning away from the source (called in [32] a shy behavior) is illustrated at left in Fig. $2 \mathrm{a}$.
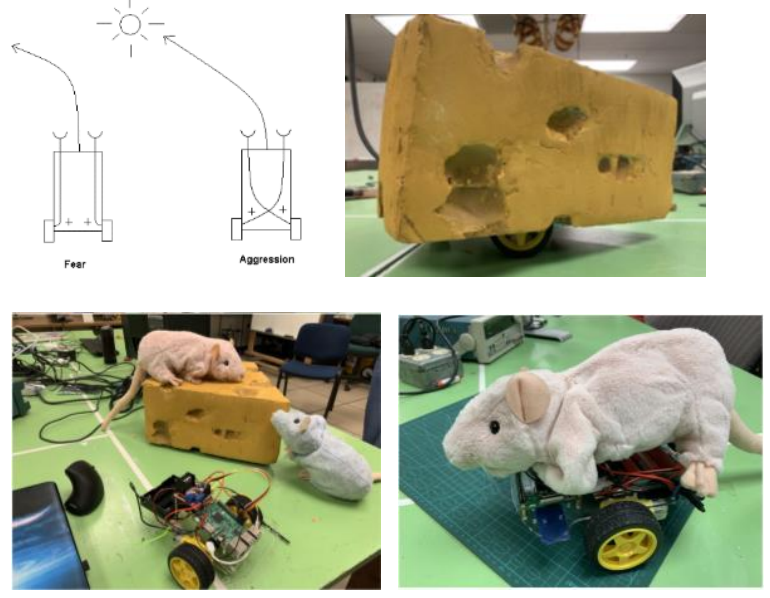

Fig.2.(a) Left Vehicle avoids light while right vehicle follows light.(b) A cheese robot, (c) red and blue mice and the base robot, (d) blue mouse. 
We can observe another type of vehicle, type (2) vehicle with positive motor connection. No change if the light is straight ahead, a similar reaction as seen in type (1). If it is to a side, then we observe the change. Here, the vehicle will turn towards the source and eventually hit it. As long as the vehicle stays in the vicinity of the source, no matter how it stumbles and hesitates, it will hit the source frontally, in the end. If the two vehicles are let loose in an environment with sufficient stimulus sources, then their characters emerge. Their characters are quite opposite. The type (1) vehicle with positive connection will become restless in their vicinity and tends to avoid them, escaping until it safely reaches a place where the influence of the source is scarcely felt. The feeling of fear is attributed [32] to this vehicle. Vehicle of type (2) with positive connection turns towards the source of light. It resolutely turns towards them and hits the source with high velocity, as if it wanted to destroy them. The aggressive feelings displayed clearly. It is really amazing to observe complex behaviors of these simple robots and their teams. The chasing or escaping actions attributed to the state of the hybrid automaton can be executed in different ways resulting from parameter values of differential equations solved in these internal states and used to generate motions.

By controlling individually the left and right wheels with velocities $\boldsymbol{v}(\boldsymbol{t})$ being solutions to different SDS (as discussed in sections II, III, and IV) we create a high variety of robot behaviors. Braitenberg Vehicles have the so-called "cart kinematics", the most common and the easiest kinematics used in small robots. In such cart robots if the same velocity is given to both wheels robot drives forward, if a higher velocity is given to the right wheel the robot keeps turning left, if the higher velocity is given to the left wheel the robot keeps turning right. If the left wheel does not move and the right wheel turns forward the robot turns around the center being the left wheel. If the right wheel turns with velocity $v$ and the left wheel turns with velocity $-\boldsymbol{v}$ the robot rotates counterclockwise around its center of gravity. It was experimentally observed that, as explained in simple examples above, with sequences of even just constant value velocities, many interesting "mobile robot gaits" are created from their simple combinations [12]. When two sinusoids are given to left and right wheels the walking pattern is created, like using left and next right leg with body rotations. When the velocity of every wheel (Fig.1) varies individually with added noise, the robot can move in very many stochastic patterns.

Noise-like processes (like Perlin or Diffusion Drift) can be added on top of any deterministic motion generations. For instance, Fokker-Planck model has parameters like $D, v$ and $w$ of equations, and the animator or the supervising software can experiment with their values. Natural noise or mechanical disturbances are simulated with $\boldsymbol{w}$. These values can be found using Nature-Inspired Methods as well [14]. In another example, in one of experiments with another robot a motion was developed for a golf-playing robot arm but next applied to the neck-moving behavior of a humanoid - interesting and unexpected gesture was thus created. We found this "general motion transfer" property to be useful in several robot theatre applications. It was observed that the motions transferred from area to area and from robot to robot can be unexpected, interesting and innovative.

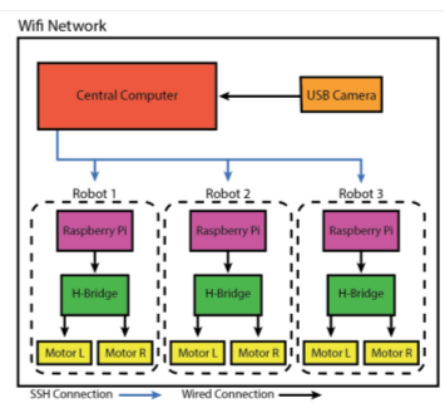

Figure 3. The Mice and Cheese System with ceiling camera and Wifi Network.

\section{B. The Mice and Cheese Chasing Game}

Fig. $2 b$ presents the robot cheese, Fig. $2 c$ two mice and the base mobile robot and Fig. $2 \mathrm{~d}$ the blue mouse robot. These are the actors of our "game theatre" - in addition to moving the robots talk to represent their emotional states such as fear. These are all Braitenberg Vehicle robots playing the roles of two mice (blue and red) that chase the cheese robot in a stochastic game of "policemen and thief" type. Chasing games and fighting games are entertaining, like in a puppet theatre. The environment in this project is a large triangle decomposed to small triangular grids with only one escape door for the cheese. The robots start from random location each in a limited triangular grid. The cheese wins when it escapes from the game area through the door. The mice win when they catch the cheese; it means they surround it in such a way near the wall that the cheese cannot move to any neighbor triangle. Each mouse has collaborative motives (catch the cheese) and egoistic motives (being first to catch it). For the lack of space we do not explain the game algorithm or technical aspects of robots. Fig. 3 presents the entire system with the ceiling camera that permanently monitors the state of game and informs the control computer. The colors of the robots are for the ceiling camera to recognize robots' positions. Behaviors of robots are based on hybrid automata. The automata internal states correspond to Fokker-Planck equations with various parameters. With parameters $\mathbf{D}=\boldsymbol{w}=0$ the automaton and the game are deterministic. However, with $\boldsymbol{w} \neq 0$ the game is stochastic and more difficult, but also more interesting to play and observe. Mice and cheese behave differently at every time. We treat this game as a simple example of a game-based improvisational, interactive robot theatre. Next section presents details of our realization. In this model each of the two wheels is controlled by a single-variable differential equation (the third wheel of the Braitenberg Vehicle is not controlled).

\section{Fokker-Planck Equation for motion control.}

An exact formalization of reliable behavior in the presence of stochasticity that unfolds in time and in space requires the model to be a dynamical system, contain stochasticity, have a continuous state space, and evolve in continuous time. The simplest possible system with all these features is a stochastic dynamical system with Brownian noise.

$$
d x(t)=v(x) d t+\sqrt{ }(2 D) d w
$$

where: $\boldsymbol{x}$ is a one-dimensional state space, $\boldsymbol{D}$ is the diffusion constant for Brownian noise $\boldsymbol{w}, \boldsymbol{v}(\boldsymbol{x})$ is called the drift coefficient and can depend on the state space. If one sets $\mathrm{D}=0$, the noise term vanishes and what one gets is a deterministic nonlinear dynamical system 


$$
d x(t)=v(x) d t
$$

While this connection with deterministic dynamical systems make the system appealing, it is not clear how to perform an analysis of such a stochastic evolution. In order to do this, one needs a mapping to a more rigorous formulation in terms of measure theoretic probability [28]. In that formulation what is more natural is the notion of Markov processes. Markov processes are stochastic processes that satisfy the following independence relation

$P($ past, future $\mid$ present $)=P($ past $\mid$ present $) P($ future $\mid$ present $)$

and can also be rewritten as

$$
P(\text { future } \mid \text { present }, \text { past })=P(\text { future } \mid \text { present })
$$

making it a statement about what knowledge is required to adequately albeit probabilistically know the future of the system at any given present moment. From this point of view, ordinary differential equation based dynamical systems are Markov processes. Both equations (3) and (4) can be made precise in measure theoretic terms and can be rewritten for any stochastic evolution on any kind of state space. In particular, when the state space is discrete and finite and the evolution is discrete. It becomes a discrete time Markov chain (DTMC) whose evolution is uniquely determined by the transition matrix

$$
T_{i j}=P(j \mid i)
$$

where $\boldsymbol{P}(\boldsymbol{j} \mid \boldsymbol{i})$ is the probability of making a transition from state $\boldsymbol{i}$ to state $\boldsymbol{j}$ at any given discrete time instant. For simple state spaces like state spaces on real line $\boldsymbol{R}$ and line segment $[0,1]$, it is equivalent to the following partial differential equations (PDE) below. Equ. (6) is example of PDE defined by Fokker-Planck operators.

$$
\frac{\partial c(x, t)}{\partial t}+v \frac{\partial c(x, t)}{\partial x}=D \frac{\partial^{2} c(x, t)}{\partial x^{2}}
$$

where $c(x, t)$ is the probability density of finding the system in state $\boldsymbol{x}$ at time $\boldsymbol{t}$. Note that this PDE is deterministic, even though the underlying system is stochastic. This equation is the classic diffusion equation originally constructed to model evolution of heat conduction in extended finite temperature materials and is identical to Schrödinger's equation known from Quantum Mechanics. It captures probabilistic evolution of particle in the absence of drift $\boldsymbol{v}$. Just as ordinary DE requires some initial (boundary) conditions to make them completely and uniquely specified, PDEs require boundary conditions. Taken together, the equation encodes all the knowledge one can acquire about the system. In classical mechanics [20], the Lagrangian $\boldsymbol{L}(\boldsymbol{q}, \boldsymbol{q})$ is defined as a function of $\boldsymbol{x}$ and $\dot{\boldsymbol{x}}$, the position and velocity of the system. In an equivalent Hamiltonian formulation, the Hamiltonian $\boldsymbol{H}(\boldsymbol{p}, \boldsymbol{q})$ is defined as a function of $\boldsymbol{x}$ and $\boldsymbol{q}$, the position and momentum of the system. An important observable in SDS and stochastic processes in general is first-exit time, the time it takes for the state of a system to cross a certain threshold. In higher dimensions, it makes sense to think of exit from a domain with domain boundary. One can associate with first-exit, a probability distribution function for the first-exit time or firstpassage time (FPT), a random variable in this system.

$$
u(x, T)=P\{\tau<T \mid \mathbf{x}(t=0)=x\}
$$

where $\boldsymbol{u}(\boldsymbol{x}, \boldsymbol{T})$ is the cumulative probability of a particle starting at $\boldsymbol{x}$ to reach the boundary before time $\boldsymbol{T} . \boldsymbol{D}$ is the domain and $\boldsymbol{B d}(\boldsymbol{D})$ its boundary. The associated random variable is represented by $\tau$. $\boldsymbol{L}$ is the Fokker-Planck operator. The associated PDE for the cumulative probability is given by

$$
\begin{aligned}
& \frac{\partial u(\mathbf{x}, t, T)}{\partial t}+\mathcal{L} u(x, t, T)=0 \quad \text { for } \quad x \in \mathcal{D}, t<T \\
& u(x, t, T)=1 \quad \text { for } \quad x \in B d(\mathcal{D}), t<T \\
& u(x, t, T)=0 \text { for } \quad x \in B d(\mathcal{D})
\end{aligned}
$$

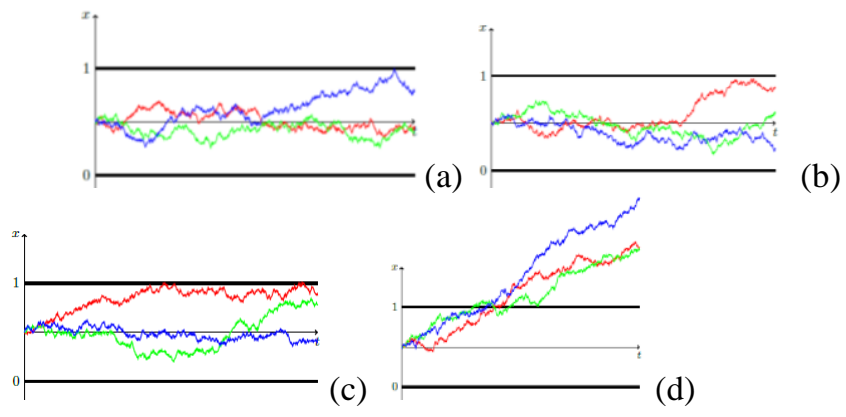

Fig.4. (a) Drift Diffusion Model (DDM) with no drift and small diffusion constant (b) DDM with very small drift and small diffusion constant, (c) DDM with small drift and larger diffusion constant, (d) DDM with large drift

Fig. 4 presents some basic examples of typical paths of SDS with constant diffusion coefficient and constant drift. The boundary points are 0 and 1 . As the velocity (drift constant) increases, one can see that almost all paths exit boundary point 1 . This is in contrast to the case where drift is zero. First-exit problems and analysis surrounding them are useful ways of thinking about transient nature of stochastic processes and have found their use in the sciences and in engineering disciplines [24]. In many nonequilibrium systems, traditional equilibrium analysis washes away details about non-asymptotic dynamics and patterns. In this paper, we identify transients and their timescales as ways to characterize biological reliability. In robotics terms one can think about ordinate as time axis and abscissa (denoted as $\boldsymbol{x}$ in Fig. 4) as a distance, velocity, radial velocity or rotation angle; the choice depending on robot design, kinematics and system of equations. Velocities from Fig. 4 are added to the fixed velocities of each wheel individually to create various gaits, behaviors and transitions to the neighbor triangular grids of the game.

\section{CONCLUSION}

As a part of a larger project "teaching by games and improvisational robot theatres" in this paper we sketched an innovative general methodology to create motions for all kinds of assistive and theatrical robots. It is based on the concept of universal motion processes that exist in Nature and are described by Fokker-Planck differential equations. Introduced robot motions generalize physiology and lift it to the systems level. In particular, by noting the surprisingly deterministic behavior of physiological processes the concept of reliable behavior is generalized and the models of nano and micro physiology, as well as atomic forces, are 
lifted to the systems level and applied to "fairy-tale" robot programming - visualization of various dynamical processes. The concepts of stochastic differential systems and first-passage time to a boundary were illustrated for mobile robots and used to generate "biological" patterns of individual and swarm motions and behaviors with new applications in robotics, such as generating a variety of gaits for Braitenberg Vehicles and robot games.

The system approach in this paper has two aspects: (1) We designed a game for three robots and observed various interesting special cases of behaviors that result from unpredictable behaviors of diffusion models as well as theatrical values of generated motion patterns. (2) We discuss with young students the mathematical and system-level questions related to the design of robot motions that allow us to show the uniformity of several processes in the world and also, how to apply system reasoning to practical problems. The role of analogy and general patterns of motion is emphasized.

\section{Future works.}

(1) In quantum mechanics, the Euler-Lagrange PDE of FokkerPlanck is replaced by the Schrödinger equation where the evolution of wave function is determined by the Hamiltonian operator. The Hamiltonian is the generator of probabilistic evolution. Nano-scale Quantum Robots are presented in [31] and Quantumly-Controlled Braitenberg Vehicle robots are discussed in $[36,39]$. Combining the quantum automata with Schrödinger equations we will be able to create new types of "quantum motions" that will generalize motions from this paper.

(2) In entertainment robotics, like here, the evaluation of Human-Robot-Interaction quality is subjective. Thus special procedures and methodologies have been developed to evaluate HRI [12]. We plan to apply them to similar "quantum biological motions" in the future.

\section{REFERENCES}

[1] B. Gros, Digital Games in Education: The Design of Games-Based Learning Environments, Journal of Research on Technology in Education, 2007, 40(1), pp. 23-38.

[2] M. Prensky, (2001). Digital game based learning. New York: McGraw Hill Press.

[3] K. Squre, (2005). Game-based learning: Present and future state of the field. Madison, TI: University of Wisconsin-Madison Press.

[4] K. Johnston, 10 Theatre Games Perfect For Drama Class, https://theatrenerds.com/10-theatre-games-perfect-drama-class/

[5] Drama Games for Kids, https://www.bbbpress.com/dramagames/

[6] Lecture By Professor Marek Perkowski - Hosted By Bytes of kitkatshttps://search.myway.com/search/video.jhtml?n=7876328d\&p2= $\% 5 \mathrm{EY} 6 \% 5 \mathrm{Exdm} 277 \% 5 \mathrm{ETTAB} 03 \% 5 \mathrm{EUS} \& \mathrm{pg}=$ video\&pn $=1 \& \mathrm{ptb}=13 \mathrm{C}$ A0213-C765-4115-B916-FC7856E87135\&qs=\&searchfor= Perkowski+robot+video\&si=EAIaIQobChMI4N6Az8at7gIVryxCh16KAfOEAEYASAAEgKu6_D_BwE\&ss=sub\&st=tab\&tpr=sbt\&trs $=\mathrm{mv} 3$

[7] Social robots for autism education, https://www.ucl.ac.uk/ioe/researchprojects/2020/sep/social-robots-autism-education

[8] D. Xu, D. Blank, and D. Kumar, Games, robots, and robot games: complementary contexts for introductory computing education, GDCSE '08: Proc. 3rd Intern. Conf. on Game development in computer science education, Febr. 2008, pp. 66-70, https://doi.org/10.1145/1463673.1463687

[9] C. Barker, (2010) Theatre games: A new approach to drama training, books.google.com https://www.google.com/books/ edition/Theatre Games/qIlhT6Bu7YC?hl=en\&gbpv=1\&dq=theatre+ga mes+in+education \&pg $=$ PR $11 \&$ printsec $=$ frontcover
[10] M. Jeon, M.F. Hosseini, J. Barnes, Z. Duford, R. Zhang, J. Ryan, and E. Vasey, Making live theatre with multiple robots as actors bringing robots to rural schools to promote STEAM education for underserved students,

https://ieeexplore.ieee.org/abstract/document/7451798?casa token=zZyr 7W2aWaYAAAAA:0 Me4LEAahZCXn3eh7Opj4wavVRBUvihgAZkg T2V3O130O57UcbiuE4AqR2BJEzXEwM2uOgxw

[11] S.Shettleworth, Cognition, Evolution, and Behavior. Oxford Univ. Press, 2010.

[12] M. Sunardi, Synthesis of Expressive Behaviors for Humanoid Robots, $\mathrm{Ph} . \mathrm{D}$. Dissertation. ECE PSU, June 2020.

[13] B. Webb. Chapter 1: Using robots to understand animal behavior volume 38 of Advances in the Study of Behavior, pp. 1-58. Academic Press, 2008.

[14] A. Slowik, H. Kwasnicka, Nature inspired methods and their industry applications - Swarm intelligence algorithms, IEEE Transactions on Industrial Informatics, Vol. 14, Issue 3, pp. 1004-1015.

[15] R.A. Brooks. Intelligence without representation. AI, 47(1-3):139-159, 1991.

[16] T. Bullock. How do Brains Work?: Papers of a Comparative Neurophysiologist. Contemporary Neuroscientists. Birkhauser Boston, 2013.

[17] J. Cartailler, Z. Schuss, and D. Holcman. Geometrical effects on nonlinear electrodiffusion in cell physiology. J. Nonlinear Sci, 27(6):1971-2000, Dec 2017.

[18] Y. Forterre, J. Skotheim, J. Dumais, and Lakshminarayanan Mahadevan. How the venus flytrap snaps. Nature, 433(7024):421, 2005.

[19] J. Gjorgjieva, G. Drion, and E. Marder. Computational implications of biophysical diversity and multiple timescales in neurons and synapses for circuit performance. Current Opinion in Neurobiology, 37:4452,2016 .

[20] K. Perlin, "An image synthesizer," ACM Siggraph Computer Graphics, vol. 19, no. 3, pp. 287-296, 1985

[21] S. Grillner and A.M. Graybiel. Microcircuits: The Interface Between Neurons and Global Brain Function. Dahlem workshop reports. MIT Press, 2006.

[22] D.R. Hofstadter and Fluid Analogies Research Group. Fluid Concepts and Creative Analogies: Computer Models of the Fundamental Mechanisms of Thought. Penguin Press Science Series. Penguin Books, 1998.

[23] A. Bruderlin and L. Williams, "Motion signal processing," in Proceedings of the 22nd annual conference on Computer graphics and interactive techniques - SIGGRAPH '95, 1995, pp. 97-104, ISBN: 0897917014.

[24] M. Unuma, K. Anjyo, and R. Takeuchi, "Fourier principles for emotionbased human figure animation, "in Proc.eedingsofthe 22 ndannual conferenceon Computer Graphics and interactive techniques, 1995, pp. $91-96$.

[25] J.H. Holland, K.J. Holyoak, R.E. Nisbett, and P.R. Thagard. Induction: Processes of Inference, Learning, and Discovery. MIT Press, 1989.

[26] R. Venkatachalapathy, Systems Isomorphisms in Stochastic Dynamic Systems, PSU, Systems Science, Dissertation, September 2019

[27] K. Immelmann, C. Beer. A Dictionary of Ethology. Harvard Univ Press, 1989.

[28] O. Kallenberg. Foundations of Modern Probability. Probability and Its Applications. Springer New York, 2002.

[29] R. Laban and F. C. Lawrence, Effort: Economy of human movement. Macdonald \& Evans, 1979.

[30] P. Langley, H. Simon, G. Bradshaw, and J. Zytkow. Scientific Discovery: Computational Explorations of the Creative Processes. MIT Press, 1987.

[31] P. Benioff, Quantum robots and environments, Phys.Rev. A58, 893, 1998.

[32] V. Braitenberg, Vehicles, experiments in synthetic psychology, Cambridge, Mass. MIT Press, 1986.

[33] P. Paoletti and L. Mahadevan. A proprioceptive neuromechanical theory of crawling. Proc.Royal Soc. B: Biological Sciences, 281(1790):20141092, 2014

[34] S. Redner. A Guide to First-Passage Processes. Cambr. Univ. Press, 2001.

[35] M. Perkowski, T. Sasao, J.-H. Kim, M. Lukac, J. Allen, S. Gebauer, Hahoe KAIST Robot Theatre: learning rules of interactive robot 
behavior as a multiple-valued logic synthesis problem, ISMVL'05, 19-21 May 2005.

[36] A. Raghuvanshi, Y. Fan, M. Woyke, and M. Perkowski, Quantum Robots for Teenagers, Proc. ISMVL07, page 18, May 13 - 16, 2007.

[37] H.A. Simon. Models of Discovery: and Other Topics in the Methods of Science. Boston Studies in the Phil. History of Science. Springer Netherl. 2012.

[38] https://www.bing.com/videos/search?q=motion+acquisition+robot+hum anoid\&qpvt=motion+acquisition+robot+humanoid\&FORM=VDRE

[39] M. Perkowski, "Quantum Robots. Now or Never?" Main address of the conference, Proc. $5^{\text {th }}$ Nat. Conf. Inform. Techn, Gdansk, Poland, May 20-23, 2007.

[40] N. Tinbergen. On aims and methods of ethology. Zeitschrift fr Tierpsychologie, 20(4):410-433

[41] M. Perkowski, and K.Liu, Binary, Multi-Valued and Quantum Board and Computer Games to Teach Synthesis of Classical and Quantum Logic Circuits, Proc. ISMVL 2021. 
